\title{
The Informational Model of Consciousness: Mechanisms of Embodiment/Disembodiment of Information
}

\author{
Florin Gaiseanu*
}

\begin{abstract}
It was shown recently that information is the central concept which it is to be considered to understand consciousness and its properties. Arguing that consciousness is a consequence of the operational activity of the informational system of the human body, it was shown that this system is composed by seven informational components, reflected in consciousness by corresponding cognitive centers. It was argued also that consciousness can be connected to the environment not only by the common senses, but also by a special connection pole to the bipolar properties of the universe, allowing to explain the associated phenomena of the near-death experiences and other special phenomena. Starting from the characteristics of this model, defined as the Informational Model of Consciousness and to complete the info-communication panorama, in this paper it is analyzed the info-connectivity of the informational system with the body itself. The brain areas where the activity of each informational component are identified, and a definition of consciousness in terms of information is proposed. As the electrical connectivity by means of the nervous system was already proved, allowing the application of the analysis and developing tools of the information science, a particular attention is paid to the non-electrical mechanisms implied in the internal communication.

For this, it is shown that the key mechanisms consists in embodiment/disembodiment processes of information during the inter and intra communication of the cells. This process can be modeled also by means of, and in correlation with specific concepts of the science and technology of information, referred to network communication structures, and is represented by epigenetic mechanisms, allowing the acquired trait transmission to the offspring generation. From the perspective of the informational model of consciousness, the human organism appears therefore as a dynamic reactive informational system, actuating in correlation with matter for adaptation, by embodiment/disembodiment processes of information.
\end{abstract}

Key Words: consciousness, informational model, matter-related information, info-embodiment/disembodiment mechanisms, epigenetic trait transmission

DOI Number: 10.14704/nq.2019.17.04.2009

NeuroQuantology 2019; 17(04):01-17

\section{Introduction}

Consciousness is still a debated topic because not all of the associated properties are understood and even less its nature. Actually, the living itself and its origin is not still understood. Both the psychological and the parapsychological properties of mind cannot be unitary explained and a large divergence could be noted in literature on this regard. As consciousness cannot be really measured, only described, consciousness was until few decades ago the field of philosopher (Overgaard, 2017), especially because no high performance tools existing today, based on the large and deep integration of the microelectronic and microsystems technologies were available, 
NeuroQuantology | April 2019| Volume 17 | Issue 04 | Page 01-17| doi: 10.14704/nq.2019.17.04.2009

Gaiseanu F., The Informational Model of Consciousness: Mechanisms of Embodiment/Disembodiment of Information

so other fields like physics and information and technology of information, besides neurology, psychology, biology were not sufficiently involved. Today, not only the cognitive and neurosciences agree that the philosophical problems of why and how there is consciousness are also their problems (Overgaard, 2017), but also psychology, psychiatry, anesthesiology, biology, genetics, geriatrics, pharmacology, and especially the physics and more recently, the science of information and technology (Gaiseanu, 2018d). We assist therefore today to an interdisciplinary effort of integration of research questions, methods and arguments.

The parapsychological properties of mind, like that exhibited during the near-death experiences (NDEs) (Fracasso and Friedman, 2011; van Lommel, 2006), showing a non-locality state of mind (Gaiseanu, 2017a), the remote communications by mind (Radin, 2006), cannot be explained by the classical laws of physics, so it was necessary to approach consciousness taking into account the quantum mechanics properties of particles and particle systems (Radin, 2006; Hameroff, 1998; Hameroff and Penrose, 2014; Shan, 2018). Even so, other categories of psychological properties of mind like the remote diagnostics (Myss, 1996), remote exploration of various geographic regions (Meijer, 2013b), and even some specific characteristics phenomena associated with NDEs like the regression to the childhood, extracorporeal view, peace and light brightness, the crossing of a "tube", or psychokinetic (anti-gravitational) phenomena (Rae et al., 1999; Radin, 2006; Radin, 2018; Bösch et al., 2006), cannot be approached only by the entanglement property.

The approach of consciousness from an informational point of view had been opening new perspectives for understanding of consciousness (Dehaene et al, 2014), starting with the analysis of the brain activity as an inter-relation between various processors (like in the computer devices) specific of conscious and unconsciousness (Baar, 1988), and continuing with a model of the perception based on a Fuzzy approach (Perlovski, 2001), with applications in artificial neural systems, and with a hierarchic entropy/informational type analysis of the integration of information by using specific statistical functions (Tononi, 2004; 2008; Tononi et al., 2016) or the mathematical theory of Kolmogorov complexity (Ruffini, 2017), to mention some of more relevant researches.
Even so, only recently consciousness was described for the first time as a global informational system of the human body (Gaiseanu, 2018d), defined as the Informational Model of Consciousness (IMC), structured in a specific seven-center type architecture and able to approach, explain and interpret in terms of information the parapsychological properties of mind (Gaiseanu, 2016a,b; 2017a,b,c), on the basis of the latest results of quantum mechanics experiments showing that the disembodiment of information from particles themselves (Gaiseanu, 2016a) is a real possibility (Aharonov et al., 2013) and of astrology (Gaiseanu, 2016a), revealing the matter/ antimatter (dark matter) structure of universe (Hajdukovic, 2013). The approach of the separation of information from physical particles (Aharonov et al., 2013) in terms of information and matter-related informational field (Gaiseanu, 2016a) and the role of dark matter as a new player in the info-panorama of the universe and consciousness (Gaiseanu, 2016a) was for the first time introduced by Gaiseanu (Gaiseanu, 2016a; 2017b,c). If today is generally admitted that information is a fundamental property of nature (Gaiseanu, 2016a; Pepperell, 2018; Meijer, 2013a; Davies, 2010), this is a consequence of previous substantial contributions on this field (Draganescu, 1990), confirmed by the further works in physics (Tegmark, 2014; Gates, 2010; Verlinde, 2010) showing that it is possible to operate with information as a physical parameter (Verlinde and thereby refs) to discover the laws of matter and of the living structures (Gaiseanu, 2016a).

Continuing this line, and observing the dual, informational (YES/NO-type) structure/behavior of universe (matter/antimatter, gravity/antigravity, entropy/anti-entropy, action/reaction, etc.), it was shown for the first time that at the global level, the human being is a bipolar info/matter structure (Gaiseanu, 2016a; 2018d), and consciousness, so information, could be disembodied from the matter support (informed matter), as a distinct entity during NDEs (Gaiseanu, 2017a; 2018a).

However, these researches are limited to the modeling of the connectivity with the body environment. Therefore, to complete the full range of the connectivity phenomena in terms of information, we have to approach also the info-transmission of the external signals inside of the body, and the implied specific mechanisms of the incorporation (integration) of information at the cell level, so the internal info-connectivity. This is a distinct task 
NeuroQuantology | April 2019| Volume 17 | Issue 04 | Page 01-17| doi: 10.14704/nq.2019.17.04.2009

Gaiseanu F., The Informational Model of Consciousness: Mechanisms of Embodiment/Disembodiment of Information

with respect to the analysis of the brain electric activity, which could be experimentally approached by highly-performant tools available now for nondestructive and non-invasive investigation, like functional magnetic resonance imaging (fMRI). Such investigations allowed to develop a series of sophisticated intelligent wireless electro-mechanic systems managed by thought, necessary to help/ improve the life quality or medical assisting/ recuperation of the users (Kaku, 2014). However, when we refer to the transmission of information inside of the human body, not limited to the nervous system, specifically to intra and inter cell communication, we have to determine what is the nature of information, what are the specific implied mechanisms and how could be possible to model such mechanisms in terms of information science. This is a necessary and justifiable effort, allowing to use finally the mathematical and analytical tools of the information science, ones demonstrated to be applicable also in this case. Moreover, this effort is also necessary in order to understand/describe/ model the complex consciousness-body relation in a unitary way and on the basis of a unitary concept (in this case information), independently of the information source. Since the antique epoch till now, this mind-body and mind-matter problem remained not understood and not solved yet (Gaiseanu, $2019 \mathrm{~b}$ ). The main objective of this paper is to reveal therefore the relation between the input information received from the environment, which is not related to the body matter (so treated as "free" information) and the matter-related form, which encodes (embodies) information, becoming finally a genetic information transmissible to the offspring. This purpose includes multi-task aspects, successively approached: (i) the analysis of the integration degree of information in interaction with matter, from free to genetically-embodied information, starting from the informational structure defined by IMC; (ii) the analysis of the implied mechanisms of intra and inter communication, their compatibility with the informational processes described by info-science, and the capability of these mechanisms to assure the transferability of the acquired information to the offspring; (iii) the identification of the phenomenological consequences derived from this analysis for the completion of the info-transmission panorama within IMC.

1. Matter-related information in the human body from the perspective of the Informational Model of Consciousness
To discuss the various levels of the matterrelated information and their relations with the informational system of the organism, we have to introduce first of all the Informational Model of Consciousness, in order to define the physics concepts and the contributing components of such a system. We have also to make a mention: when the external (electro-magnetic, mechanical, chemical) signal (agent) attains one of the sensing elements of the body (sensors), this signal starts to interact with the body matter, information becoming an information input for the informational system of the human body. As mentioned, information is proved to be closely related to the electrical signals inside of the nervous system. However, in order to include also various other specific (non-electrical) mechanisms like the physico-chemical reactions, able to embody/disembody information, we refer in this case to matter-related information as a more general concept, able to fit actually the body reality. This represents a key tool within IMC to approach the complex mind-body problem, reducing it to an information-matter interaction process. The huge quantity of information "embodied" in the living structures shows that these represent a higher level of matter-related information structures, as it was explained before in detail (Gaiseanu, 2016a), allowing a high level of reactive capacity and thus of adaptability to the environmental conditions (Kaku, 2014; Gaiseanu, 2016a). As it was previously discussed (Gaiseanu, 2018d), the quantity of information of a system is basically calculable by the difference between the entropy of the system in two distinct states (Shannon, 1948; Cover and Thomas, 1991; Gaiseanu, 2018d).

\subsection{The Informational Model of Consciousness (IMC)}

In Fig. 1 it is represented the schematic structure of consciousness, according to the Informational Model of Consciousness (IMC) (Gaiseanu, 2018d). The cognitive informational centers of consciousness shown in the right side of Fig. 1, suggestively called Icreated (Icd), Icreate (Ic), Iam (Ia), Ilove (Ia), Iwant (Iw), Iknow (Ik), Ibelieve (Ib) correspond (dashed line) with the perceived information from the associated informational subsystems managed by the brain, which are respectively: (1) the Info-Genetic Generator (IGG); (2) the Genetic Transmission System (GTS); (3) the Maintenance Informational System (MIS); (4) the Info-Emotional System (IES); (5) the Center of Decision and Command (CDC); (6) the Center of Acquisition and Storing of Information 


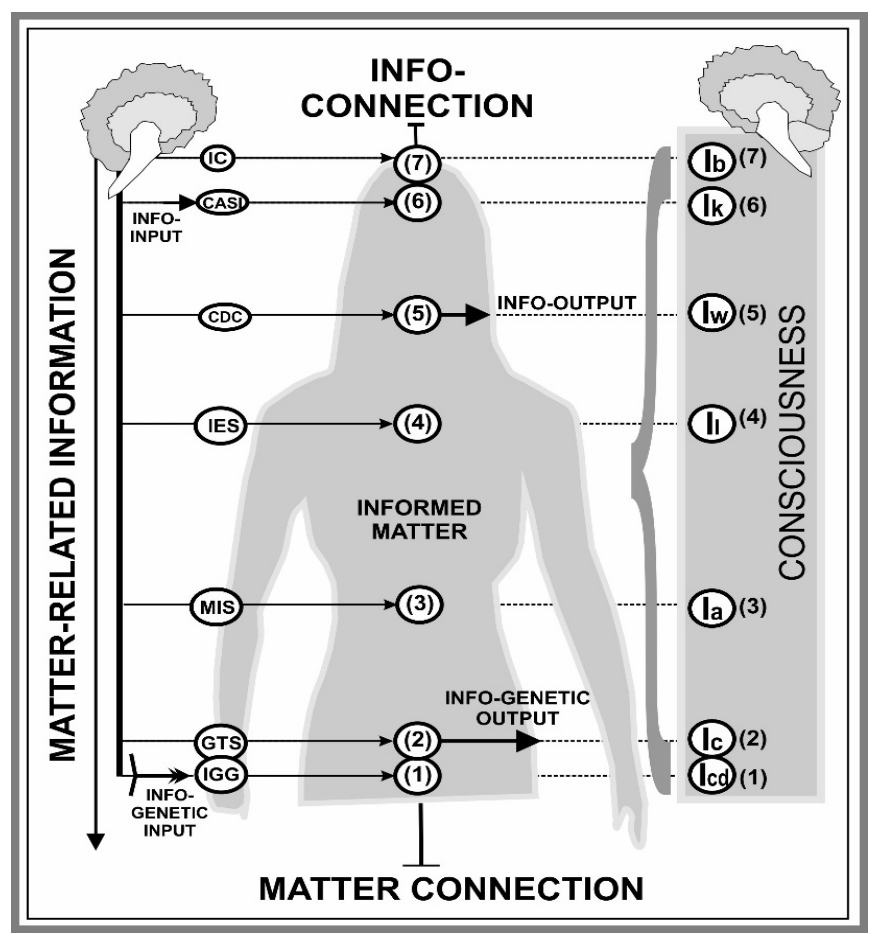

Figure 1. Schematic representation of the circuits (full lines in the left side) of the informational system and its components (marked on the connections with the body but managed by the brain), and of the corresponding consciousness components (informational cognitive centers in the right side).

(CASI); the Info-Connection (IC) pole, particularly explaining the parapsychological phenomena.

The specific definition of the cognitive centers taking into account explicitly the first person (I), is a form to admit that consciousness is a subjective, personal property (Gaiseanu, 2019b). The names of the cognition centers derive also from the main function of each of the informational subsystems which they are associated with, in a brief description as follows:

(1) IGG is the informational subsystem managing the body evolution according to the genetic input information received from the two parents and is detected in consciousness as the center Icd, expressing the inherited abilities and predispositions;

(2) GTS is the informational component responsible for the info-genetic generation/transmission, representing the info-genetic output of the body, and is related in consciousness as the Ic center, sensitive to associativity and social relations, but also with the responsibilities to form/create the new generation (Gaiseanu, 2018c,d);

(3) MIS controls automatically the information necessary to the correct and continuous functions of the organism as a whole, detected in consciousness as a cognitive center Ia (selfstatus), connecting the body (defined as informed matter) to the nourishing matter (foods, water, air) and managing the absorption/desorption processes (connection to the matter pole);

(4) IES controls the body reaction to information, especially (but not limited to) that of the hearth, which should be able to distribute the nutritive elements to the local consumers as a function of the momentary necessities, perceived in consciousness as the emotional center suggestively called Ilove (II); this name reflects the great importance of love, which is one of the fundamental state of life (Gaiseanu, 2019a 2018d);

(5) CDC is the operational and command center of information, reflected in consciousness by the decision (Iw), as an information output;

(6) CASI represents actually the data library, where it is stoked the life experiences, reflected in consciousness by memory (Ik);

(7) IC is the Info Connection pole of the organism, assuring the consciousness connection with the matter/anti-matter (dark matter) field, reflected in the cognitive center $\mathrm{Ib}$ as trust, confidence and protection (Gaiseanu, 2017a,b) and explaining in special circumstances the parapsychological phenomena (Gaiseanu, 2018d) like extracorporeal view (Gaiseanu, 2016a), the associated phenomena of NDEs (Gaiseanu, 2017a), remote transmissions by mind, and other extra-properties of the mind (Gaiseanu 2017b,c).

Fig.1 shows a schematic representation of the circuits (full lines in the left side) of the informational system and its components (marked on the connections with the body but managed by the brain), and of the corresponding consciousness components (informational cognitive centers in the right side).

From the informational point of view, the seven informational subsystems described above are distinct, butnotindependent: theyare complementary each other, and their functions are correlated to work for adaptation, each of them assisting dynamically the needs of the organism, according to the momentary or longer-time requirements. As a consequence of this correlation, the attitude, which is the dynamic output decision of CDC, is practically the result of the contribution of all other centers, or 
NeuroQuantology | April 2019| Volume 17 | Issue 04 | Page 01-17| doi: 10.14704/nq.2019.17.04.2009

Gaiseanu F., The Informational Model of Consciousness: Mechanisms of Embodiment/Disembodiment of Information

of a part of them, either as collaborative participants like IES (emotions), or providing decision criteria with respect to the specific objective, allowing an adequate selection between YES and NO command (Gaiseanu, 2018c).

The main function of CASI is to acquire and stoke information. After a certain information is acquired, this information becomes active only if it is accessed by the operator "though". In other words, not the entire information from CASI, although available, can become operable in the same time, only a certain interest information is activated according to the necessities. This operator is a vector on the informational data (field), because it needs an address and a command to go for the activation of a certain information. This dynamic operation is managed by the decisional CDC system. Therefore, while CASI accumulates "stand-by" information, like the hardware support in the informational systems, CDC is a dynamical (analytical, logical or algorithmic) operational system, managing existing information to formulate a certain decision, like in the software sequences of the informatics systems. At the end of this operational process, CDC orders to the suitable executive motor elements like muscles to apply the decision, and/or deposits the decision into CASI in a stand-by state, for later communication. That's why CDC output is mainly related to the language, as a main expressible tool of attitude, for a suitable communication process. The communication is actually a reactive process with respect to the input information, initiated by adaptation needs to own or environmental conditions.

IMC is closely correlated with neuroscience, first of all because consciousness is a result of the capture and operation of information. However, although this fact is largely accepted today by the scientific community, little attention is paid to the relevant concepts and tools deriving from the science and technology of information in the investigation of the brain functions and activities. Some relevant progresses on this field were recorded quite recently (Tononi et al., 2016), checking by fMRI experiments a postulated integration model of information earlier proposed (Tononi, 2004), but not a global multicenter informational model (like IMC) was actually proposed up to date. That's way IMC can become a challenging base of participation of all other interested sciences, specifically of neuroscience, for further and future researches on this line. This paper is circumscribed actually to such a necessary stimulating effort, bringing information in the foreground as a main and coherent concept useful for understanding and modeling consciousness.

To understand the connections of the brain with the body from the neuroscience perspective, the relation between the activity of the informational sub-systems described above and the associated areas of the brain which support their characteristic activities and functions (Hines, 2018; Baars and Gage, 2013) are mentioned as follows.

CASI is active especially in hippocampus, where the long-time memories are stoked. The short-term memory (with low storing capacity and short time (1 min.) duration) occurs in the prefrontal cortex. The skill memory, where are stored procedural learned acquired information like the playing an instrument or driving a bike, is processed in the cerebellum. Thalamus is a relay station for sensory impulses (taste, touch, heat, cold, pain, some smell) that reach cerebral cortex from spinal cord, brain stem and cerebellum. Cerebrum is the largest part of the brain and is implied in interpreting touch, vision and hearing, the main sensorial signals.

CDC is related to cerebral hemispheres $(60 \%$ of brain mass), which is the representative area of thought, creativity, communication. Particularly, the frontal and prefrontal lobes of cerebrum elaborate the thoughts, intelligence, motivation, judgement, planning, voluntary control of muscles, voluntary movement. Cerebellum, the second large part of the brain helps also to coordinate muscles, including learning and storing motor skills, as mentioned above. The left hemisphere is responsible for language and speech, comprehension, arithmetic, and writing and is the dominant hemisphere as concerns the hand use. The right hemisphere controls creativity, spatial ability, artistic, and musical skills.

IES is correlated with the limbic system (thalamus, hypothalamus, hippocampus, midbrain, amygdala), managing the emotions and all sensory impulses. MIS operates especially in medulla, which is the lowest portion of brainstem and helps the control of vital autonomic reflex functions like cardiac activity (rate and force of heartbeat, vasomotor regulation, variation of diameter of blood vessels, the distribution of blood to specific organs, blood pressure) and respiratory activity (the rate and depth of breathing). Brainstem region of brain acts as a relay center connecting the cerebrum and cerebellum to the spinal cord, performing many 
NeuroQuantology | April 2019| Volume 17 | Issue 04 | Page 01-17| doi: 10.14704/nq.2019.17.04.2009

Gaiseanu F., The Informational Model of Consciousness: Mechanisms of Embodiment/Disembodiment of Information

automatic functions such as breathing, heart rate, body temperature, wake and sleep cycles, digestion. Hypothalamus controls and integrates activities of autonomic nervous system.

GTS is correlated with hypophysis and hypothalamus, which control the sexual development and response. IGG functions are related also with hypophysis and hypothalamus, known to regulate additional important processes like growth, development, metabolism and as recently suggested, the aging (Zhang et al., 2017). Basal ganglia seems to have a role in personality features. As the individuals strongly differ in their abilities to learn specific skills, the inherited predispositions should play a determinant role. This was recently demonstrated (Herholz et al., 2016), showing that distinct regions of the brain (right auditory cortex, hippocampus, caudate nuclei) are involved in the higer performance of some participants of a piano-learning experiment. The contribution of the genetic predisposition or talent with respect to a learning obtained skill is discussed as the "nature or nurture" problem. Taking into account the sever limitations in the field of the investigation tools, new relevant evidences are expected to be obtained by the high involvement of fMRI.

According to the above description in terms of information, the human body appears in such way as a bipolar info-matter (or informed matter - IM) structure, connected both to information (field) and to (ordinary) matter. Within such a structure, one of the important cognitive center, besides Ik (memory), Iw (decision), Il (emotion), Ia (self status), Ic (genetic transmission) and Icd (genetic inherited reception) is the so-called Ib center, which is the connecting pole with information, particularly with the anti-entropic field of anti-matter (Gaiseanu, 2016a; 2017a,b), assimilated, according to some of the last discoveries in astrophysics, with dark matter (Hajdukovic, 2013). Ib could be associated with the protection feeling and with trust, specific for the religious experiences, and also with the meditation process, serving for stress relaxation, as a hypnotic-based palliative therapy (Satsangi and Brugnoli, 2018; Brugnoli et al. 2015), and as a learning and psychic reprogramming processes of mind (Dulcan, 2009). The curative role of this connection is recognized more and more by the neuroscience (Inzlicht et al., 2011), and various reports show that the religious and associated processes are active within certain areas of the brain. A quite recent study (Inzlicht et al., 2011) suggests that the religion's palliative attributes can be identified specifically in the anterior cingulate cortex.

In terms of information science (certainty/ uncertainty $<=>$ information/entropy) and according to IMC, the transition from a multi-state level of possibilities explored by the mind (i.e. from a high incertitude, expressed by a high entropy), to a certainty level represented by only one (desired) objective (so information), by means of a "collapsing" (reducing) process like in the quantum mechanics, is an anti-entropic mechanism, yielding equilibrium, peace, serenity and health (Ib). This is a powerful process, because the dissipated energy between many possibilities (uncertainty) is reduced to only one, according to the main objective, helping the achievement in a shorter time and in an effective way of this objective.

Resuming,itcanbeconcluded thatconsciousness could be described by an Informational Model of Consciousness (IMC), showing that consciousness is composed by seven informational centers, correlated with the corresponding execution elements of the body (defined as informed matter - IM), according to Fig.1. The informational connection circuits sharing/ connecting information with transducer/execution elements are formed by main spinal nervous bundles coming through the spinal cord from the brain, and the composing main execution elements as represented in Fig 1 (numbers inside of circles). This info - distribution depicts a similar structure like the empirically-detected structure related by chakras (Gaiseanu, 2018d) and described by some oriental philosophies (Ignatenko, 1994; Odour, 2014). This coincidence opens a suitable gate, based this time on the updated scientific data, for a real understanding of consciousness with respect to the empiric millenary experience of humanity, and a new line and opportunity for further researches on such a challenging issue (Gaiseanu, 2019b).

\subsection{Matter-related information within the informational subsystems}

The informational system of the organism operates for adaptation by learning. The new information is memorized by association with other older and stabilized information in Ik. For adaptation, the center Iw operates with the existing information from Ik and delivers a decision (Gaiseanu, 2019a). The decision is made on the basis of decision criteria (Gaiseanu, 2018c), associated to each informational centers: Il (emotion), Ia (self status), Ic (associativity), 
NeuroQuantology | April 2019| Volume 17 | Issue 04 | Page 01-17| doi: 10.14704/nq.2019.17.04.2009

Gaiseanu F., The Informational Model of Consciousness: Mechanisms of Embodiment/Disembodiment of Information

Icd (predispositions, aptitudes, mentality), Ik (experience), Ib (trust/certainty level) (Gaiseanu, 2018c, Gaiseanu and Graur, 2018). Therefore, the decision (expressible by attitude), could be delivered as an informational non-matter related output, and depends on the operability of all cognitive centers. On the other hand, the genetic input and output of information, controlled by IGG and GTS respectively, contain embodied (genetic) information. MIS manages the automatic processes of the recycling of matter, as a matter-related pole of the organism.

The components of the informational system of the human body act in an integrated form, information playing a role in every of its forms, from "free" to genetically embodied information. By convenience, we define as "free" the input information, because this is not yet related to the body matter. As the genetic info represents the maximum embodied degree of information, the info-integration degree should vary from free (corresponding to zero) to closely embodied information under a genetic form. Therefore, the intermediary levels of info-integration will be simply referred as matter-related information. The axis represented in the left side of Fig. 1 indicates the increase of the integration degree of information from the info-connection pole to matter connection. This axis allows a qualitative evaluation of the degree of info-integration in the components of informational subsystems, represented also in the left side of Fig. 1. Indeed, the subsystem IC operates practically with free information, marked in Fig.1 (left side) as the lowest corresponding value on the matterrelated information scale. In the subsystem CASI it is received and stored the specific information from the external and internal sensors by the informational circuits. The storing of information is a first step of information binding with matter, becoming a materrelated information in memory. However, this step assures a fragile relation, according to the short-time and large-time memory process. A large quantity of information is forgotten, remaining especially in memory only the information of interest. The repetition process of the same type of information is a basis for its integration in the informational system, triggered as a chain-type automatic prototype. Such a process would be a second step of integration of information. The subsystem CDC plays a substantial role in these operations. CDC closely works with CASI, calling the stored information and also transmitting the decision to CASI or to the execution elements, transforming it mainly into mechanical expression.
The repetition converts certain information or a chain of information in an automatic prototypes, as a higher form of the integration of matter-related information.

IES is an intermediary subsystem operating between the free - information and matter-related information, because as a general rule, the emotions, either acquired or integrated by default into the informational system, are released automatically, depending on the received information. The emotions are closely related to the execution elements, they mobilize the organism to the action, to the change of the regular, normal present state, engaging into such a process the functions of various organs of the body. The recall of some events from the past, also trigger the associated emotions, because they are also memorized in Ik, so the releasing of emotions can be consciously initiated.

MIS is a self-assisting program assuring the automatic functioning of organism especially to manage the input/output matter processes (food, water, air), but also the organizing processes of inner distribution and auto-control. Within this system, the information is highly linked to matter, in highly stabilized automatic programmed informational chains. The stereotype behavior acquired by learning is integrated in this system, detaching the mind of conscious participation and therefore minimizing the learning effort. This represents a higher degree of matter-related information of the informational system of the body.

In GTS and IGG the information is highly related to matter, prepared to be decoded during the formation of a new human being. The integration level of information within this stage is very high, practically representing the top level.

Therefore, looking to the variation of the infointegration degree from free-type information to the embodied (matter-related information) under the normal (regular) conditions, it seems that this increases from CASI to IGG, as it is shown in Fig. 1 by the axis with arrow directed to the bottom side of the figure. Taking into account such a general view, the question is how a new received information could be incorporated into the informational system of the human body, so that this information might be transmitted to the next generation, without affecting the genetic structure of the cell. With other word, the question which arises is how a new (non matter-related information) might be converted in 
NeuroQuantology | April 2019| Volume 17 | Issue 04 | Page 01-17| doi: 10.14704/nq.2019.17.04.2009

Gaiseanu F., The Informational Model of Consciousness: Mechanisms of Embodiment/Disembodiment of Information

an embodied (matter-related) information, which is to be disembodied (decoded) in a next phase of an embodiment/disembodiment process. This question is analyzed in the next section.

2. Embodiment/disembodiment mechanisms of information and phenomenological consequences

In this section will be detailed some mechanisms of transmission of information inside of the organism. In general, when we talk about the transmission of information, we refer to the nervous system. However, as it will be presented below, the cell intra and inter communication are natural and permanent characteristic processes, and the mechanisms of such a communication consists in information embodiment/disembodiment processes.

\subsection{Inter and intra-cellular mechanisms of information transmission}

As our daily activities depends strongly on electronic information-processing tools, our survival depends every moment on our biological-processing systems (Laub, 2016). Each of the trillions of cells of the human body may detect and respond to a wide range process information and make suitable decisions for adaptation. The signaling could be transmitted or received by chemical compounds (like the nutrients), electrical impulses (neurotransmitters -- inducing electrical signals along nerves and heart beats), or mechanical stimuli (touch and hearing, stretching of the stomach when it is full).

The chemical communications could take place by paracrine (within a relatively short distance), synaptic (at the junction between two nervous cells) and endocrine (large distance) signals. These kind of signals consist in molecules of proteins or other type of molecules, known under the generic name of ligands, which are produced by the sending cell and are released into the extracellular space. According to such a mechanism, only certain types of cells are sensitive to certain type of ligands, that which possess the suitable receptor bind on their surface (Albert et al., 2008; Sogin et al., 2003; Barritt, 1992; Gawad et al., 2015). This mechanism seems to be similar with the operational informational unit IF (condition) $=>$ THEN (consequence), in the computer software, determining a conditional selection process. When a receptor of the target cell binds a signaling ligands, a series of chain reactions take place into the interior of the cells, the extra-cellular signal inducing an intracellular signal, which could affect the activities of the genes. In the particular case of the transmission by a nervous cell, when a cell fires due to an input stimulus, an electric signal moves rapidly along the axon to the synapse (the junction gap between two cells), triggering the release of vesicles with ligands (in this particular case named neurotransmitters) to the neighboring target cell receptors. After this step of transmission, the sending cell "resets" rapidly in a standby state, prepared to receive another new signal. The electric signal is carried by a mechanism of the type "all or nothing", implying an operation by ion channels and electrical potential along the cellular membrane. Therefore, the nervous cell fires ("switches" to an ON state) or not (OFF state) similarly with a YES/NO informational mechanism.

The paracrine signaling is important during the growth of tissues allowing that the neighborhood cells to understand what type of identity to proliferate. Whereas paracrine communication refers to external groups of cells, the autocrine communication consists in a reception at the surface or in own bulk of some ligands produces by the cell itself. This mechanism is very important to reinforce itself correct identity, having a key role in cancer. The endocrine signaling refers to the intercellular communication by hormones, which are active substances secreted into the bloodstream to transmit signals at distance to target cells.

As we can observe, the electrical signals are characteristic for the nervous cell, but the communication between the rest of the cells and nervous cell junction is performed by molecular physico-chemical processes, so by a matter-related support. A physico-chemical mechanism sustains also the nervous cell inter-connection. Information in such type of mechanism is transferred by embodiment/disembodiment of information. These terms points out the nature support of information, distinguishing it from the electrical transmission. To concretize with an explicit example what embodiment and disembodiment mean, we can take into account one of the previously mentioned process: when a matter supported signal - initiator (for instance a ligand molecule), transfers information to another component of a system (for instance a cell surface receptor) by binding to it (physico-chemical mechanism), this corresponds with a disembodiment process of information from the initiator, and an embodiment process of information by the receiver. Therefore, the information is transferred by sequential embodiment/disembodiment steps, 
NeuroQuantology | April 2019| Volume 17 | Issue 04 | Page 01-17| doi: 10.14704/nq.2019.17.04.2009

Gaiseanu F., The Informational Model of Consciousness: Mechanisms of Embodiment/Disembodiment of Information

either inside or outside of the cell. As this type of mechanism is typical for the cell communication, this will be tacitly assumed everywhere it is the case. The same suggestive term was also used within a quantum mechanic experiment, showing that information (Gaiseanu, 2016a) can be disembodied from particles (body) themselves, as a distinct entity (Gaiseanu, 2016a; Aharonov et al., 2013).

These mechanisms take place basically by a YES/NO type action. In other words, the signal coming from the upstream molecules turns $\mathrm{ON}$ (activates) a specific signal in the target, passing then in a downstream and turns OFF (inactivates) (Asashima, 2010), similarly with a YES/NO process in the computing systems. The cellular communication plays a fundamental role in the biological life for the maintenance of the functions of the organism under normal conditions, as a whole. This communication allows actually the adaptation of the cells to the conditions of the extracellular environment, controlling the development, growth, immunity, the regulation of the metabolism, altering or maintaining the differentiated state, determining whether the cell should divide, or if it will live or die. The errors of communications may result in cancer growth and diabetes (Gawad et al., 2015).

The intracellular communication pathways can be actually regarded as an interconnected network where the signals are processed along multiple parallel routes of interaction, like in the computer-based networks, known as neural networks (Sogin et al., 2003). This is an example of collaboration between the information science and life and neuro-science to understand better how nerve cells distribute and process information in the brain, which can be correlated with the properties of the intracellular signaling. Following this line of correlation, the connections between the computer units in the neural networks could be considered analogous with the synapses in the nervous system, so the cell signaling network can be viewed as a neural network, able for adaptation processes.

\subsection{Epigenetic mechanisms for trait transmission by embodiment/disembodiment of information}

The new characteristics (traits) acquired during the life of a person could be transmitted, according to such a described process, to the descendants. Although this affirmation was and is supported by general empirical observations, only relatively recently were registered real progresses in understanding of the implied mechanisms of such form of transmission, especially based on the decisive role of the high-tech instrumentation assisted by the microelectronic/ microsystems as intelligent and/or sensitive (sensor) devices, permitting a deep analysis of the cell and cell systems. Such a transmission, studied by a relatively new science, i.e. epigenetics (Shelley et al., 2009), is performed in such a manner that the genetic structure is not modified at the cellular level, however the acquired information is distinctively reproduced by the subsequent generations of cells. Concisely, the epigenetic science is dedicated to the study of the heritable traits that do not depend upon the primary sequence of DNA (deoxyribonucleic acid) (Gorski, 2013). An epigenetic trait is a stably heritable phenotype (observable properties of an organism produced by the interaction with the environment), resulting from changes of the chromosome without alterations in DNA sequence (Shelley et al., 2009). The genotype is the genetic constitution of an individual organism, expressed by the particular type and arrangement of genes. To operate with these concepts, we have to note that the chromosome is a structure in all living cells, consisting of a single molecule of DNA (double helix structure) bonded to various proteins (histone molecules anchoring it) and that carries the genes determining the heredity. The eukaryotic cells are the cells of the evolved organisms, basically including the plants and animals, composed by nucleus and organelles, enclosed by a plasma membrane, contrarily to the prokaryotic unicellular organisms, which do not have a membrane.

The basic epigenetic mechanisms could be discussed taking into account three main categories of signals, which culminate in the establishment of a stable heritable epigenetic state (Shelley et al. 2009), as presented below.

(A) The "epigenator" is a signal emanated from environment and which is able to activate/initiate/ trigger the subsequent intracellular pathway category of signals, defined in (B) as "initiator". As a signal (information, according to IMC), the epigenator could be a protein-protein interaction, or a modificationbased event (matter-related information, according to IMC) which could trigger a second internal signal, that of the "initiator" (explicated in item (B)). One of the most important characteristics of the epigenator is that this info-signal should persist in the cell for an enough long time, in order to be able to unleash the epigenetic phenotype processes, although it is not necessary to remain inside of the cell during the 
NeuroQuantology | April 2019| Volume 17 | Issue 04 | Page 01-17| doi: 10.14704/nq.2019.17.04.2009

Gaiseanu F., The Informational Model of Consciousness: Mechanisms of Embodiment/Disembodiment of Information

subsequent events step (Shelley et al., 2009). From the IMC perspective, this characteristic could be clearly associated with the repetition process invoked by IMC, as an imprescriptible condition at the macrolevel of the organism, to acquire an automatic, stable trait. At the cellular level, we have to note that during this step, the epigenator disembodied the external information, transferring it to the initiator, which embodied the received information, as mentioned above.

(B) The epigenetic "initiator" is the informational local intracellular response of the chromatin to the "epigenator" info-signal. To operate with specific terms, we have to remember that the chromatin is the material composing the chromosomes, and consists of a complex of DNA (30-40\%), RNA (ribonucleic acid, 1-10\%) and proteins $(50-60 \%$, generally termed histones), assuring the dense package of DNA structure to prevent its damages, and playing an essential role for the DNA reinforcement during the cell division and the regulation of the gene expression and DNA replication. The proportion of these components varies not only for different species and for various tissues of the same organism, but even in the same cell, depending on the various stages of the cell cycle. The initiator defines the location on a chromosome where the epigenetic chromatin state should be established (Shelley et al., 2009), and could be a DNA-binding protein, a noncoding RNA, or any other entity (matter-related info, according to IMC) that can define the coordinates of the chromatin structure to be assembled. The initiator info-signal disembodying the carried information, may be self-reinforced or self-renewed through positive (self-amplified) feedback mechanisms, like in the electronic processes. From the operational point of view, it is sufficient that the initiator to induce in the cell a phenotype process and remain inside of the cell together with the "maintainer" (described below).

(C) The epigenetic "maintainer" signal (embodied information, according to IMC), sustains the induced modifications in the first and subsequent generations. This step consists in various pathway forms, like DNA methylation (DNA methylation is the substitution of a methyl $\left(-\mathrm{CH}_{3}\right)$ group to the carbon atom in position 5 of a DNA segment), histone modifications and variants (most epigenetic interventions occur on the histones that coat/anchor DNA), nucleosome positioning, chromosome coating with (long) noncoding RNAs, and others (Shelley et al., 2009; Cortini et al., 2016). We have to remember that a nucleosome is a structural unit of a eukaryotic chromosome, consisting in a finite length of DNA, coiled (anchored) around a core of histones (Alberts et al., 2008). The maintainers dispose of the common property that they can operate at any chromosomal location to which they are recruited by the initiator (Shelly et al., 2009). They carry the acquired embodied information during the cell cycle or in the differentiated cell types. On such a way, the cells may keep the memory of the info intervention and embodied from their ancestors to their descendants, allowing that cells with the same DNA structure to have distinct properties. These properties are stable over time and are transmitted by cell division.

From the chemical point of view, these mechanisms seem to be quite diverse and complicated. However, analyzing some mechanisms from the physics point of view, some common properties could be distinguished and treated by mathematical simple models, extracting useful conclusions in terms of information. For instance, the epigenetic feedback is referred, like in certain electronic circuits, to a further amplification of a signal output, starting from a certain value, as a consequence of its additional contribution to the input signal. In epigenetic terms, this can be resumed as follows: when the expression level of a gene becomes sufficiently high, the epigenetic feedback regulation is achieved, which further activates the expression and vice versa (Furusawa and Kaneko, 2013). Moreover, the activity of the epigenetic feedback regulation and of gene expression dynamics depends on the cellular activity, expressed for instance by the growth rate (Furusawa and Kaneko, 2013). As a consequence, it can be defined a "threshold" of such a reaction, by means of a regulatory factor, when this rate could rapidly increase to the maximum value 1 (full expression), or rapidly decrease to 0 , i.e. the state of non-expression (Furusawa and Kaneko, 2013). In terms of information, this is actually an informational YES/NO-type bistable mechanism. In such a dynamic process, gene expression in a cell refers to the synthesis of the corresponding protein, which may affect the expression of other genes, thus giving rise to a regulatory network (Furusawa and Kaneko, 2013). To clarify the operating concepts, we have to remember that gene is a specific functional unit sequence on the chromosome of DNA or RNA, able of inheritance transmission and expression of one or more traits to the offspring. Furthermore, 
NeuroQuantology | April 2019| Volume 17 | Issue 04 | Page 01-17| doi: 10.14704/nq.2019.17.04.2009

Gaiseanu F., The Informational Model of Consciousness: Mechanisms of Embodiment/Disembodiment of Information

an epigenetic trait is a stably heritable phenotype resulting from changes in a chromosome, without alterations in the DNA sequence, the main role of DNA being to encode the genetic instructions required to synthesize proteins, the basic "bricks" that ensures the proper functioning of the cell (Cortini et al., 2016).

Analyzing therefore the mechanisms involved in the initiation, spreading, maintenance and heritability of epigenetic operational ("marks") signals, there were observed in vivo a few common characteristics of them: (a) the multi-stability: (b) their spatial patterns (3D structuration); (c) their heritability. Epigenetic marks are chemical covalent modifications involved in the basic epigenetic mechanisms, either DNA (DNA methylation) or histones modifications (Cortini et al, 2016), as the maintainer signals. The bistability (so the informational basic mechanism) is therefore a general property of the epigenetic processes, the epigenetic marks acting as switches YES/NO between different functional states. In the simplest case, different patterns of epigenetic marks allow to switch between two states that have a well-defined functional characterization (bistability). Such functional states are then inherited by the daughter cells, which is what we call heritability: (Cortini et al., 2016).

During the growing process, the stem cells differentiating process, depending on the sort of tissue, implies also epigenetic mechanisms. During their development, including the initiation, stabilization and propagation stage of the patterns of gene expression, the stem cells are able of differentiating to the desired fate, through dramatic epigenetic modifications, becoming finally stabilized and heritable (Cortini et al., 2016). Epigenetic marks actually act as "switches" between different functional states, like in computers, where the switching operation is performed by some specialized integrated components (commutation transistors). The reduction of the possible (multi)states of uncertainty, to one certain state, by means of such physico-chemical mechanisms, is actually a process of information embodiment, and the quantity of information integrated into the system can be expressed by a difference of entropy between the two different situations (Tononi, 2008; Gaiseanu, 2018d). Marking a new progressive step in the understanding how epigenetics controls some functions of the living structures, a 3D bistability model of threestate was successfully adapted to the description of the vernalization, which is a mechanism allowing plants to flower after a prolonged cold period. This was especially possible by a suitable description of the temperature-dependence of the cell epigenetic bistable reactions (Cortini et al., 2016; Dodd et al., 2007).

The epigenetic mechanisms become interesting themes of researches for the understanding of some specific processes involved in the long-time memory formation and some disorders in the cognition processes in the adult, like RubinsteinTaybi syndrome, Rett syndrome, Fragile X mental retardation, Alzheimer's disease, Schizophrenia, in which observable derangements and dysfunctions in molecular components of the epigenetic apparatus have been implicated. Among these, schizophrenia is a serious disorder of cognition, which is manifested by inability of normal behavior in social situations and in performing everyday cognitive tasks (Levenson and Sweatt, 2005). Following such investigations, a rapid growing literature on this line brings new proofs of the implication of the epigenetic mechanisms both in the long-term (consolidated) memory (Ludin et al., 2011; Jarome and Lubin, 2014) and in the dysfunction of the epigenetic processes in diseases like anxiety, autism, depression, epilepsy, substance abuse.

Starting to burgeon in the 2000s years, the discipline of epigenetics allows the opening of novel perspectives for the understanding of learning and memory, and for the first time, memory was proposed to depend on epigenetic mechanisms (Bisrat and Mansuy 2017). The repetition process invoked by IMC to consolidate the new information in memory is supported by the recent discoveries, which show how are formed the memories and how the learning is reinforced by repetition, converting the labile short-term memory state to a stable longterm memory state, through a process referred to as a memory consolidation (Jarome and Lubin, 2014). The new growing field of "neuroepigenetics" is basically supported by the study of epigenetics at brain level, showing that some memories simultaneously reconsolidate in multiple brain regions, i.e. amygdala, hippocampus, and anterior cingulate cortex after retrieval (Jarome and Lubin, 2014). The ensemble of the data collected so far demonstrates that epigenetic mechanisms are engaged as integral molecular components of memory formation and are particularly important for long-term memory (Bisrat and Mansuy 2017). 
NeuroQuantology | April 2019| Volume 17 | Issue 04 | Page 01-17| doi: 10.14704/nq.2019.17.04.2009

Gaiseanu F., The Informational Model of Consciousness: Mechanisms of Embodiment/Disembodiment of Information

2.3 Informational modeling of cell and cell network communication

As the intra and extra signaling of the cells are actually parts of a communication process, this could be analyzedbythetools of theinformationand technology of information, as defined earlier (Shannon, 1948), and interesting results were recently reported (Faria et al., 2014). In a communication process, similarly as in the microelectronic systems, the communication participants are: the source (transmitter), a receiver, a transmission channel and a coder/decoder system. In the biological communication systems, the messages are transmitted and received such that the cellular machinery realizes the processing of these messages according to its needs. Inside of the cell, the transmitter is assimilated with nucleus, where the information is stored/generated in DNA and transmitted by RNA. In a communication electronic system, the receiver consists of the blocks demodulator/decoder where the information is received, while in an eukaryotic cell, this role is played by organelle, where the information (protein) is received (Faria et al., 2014).

Starting from the observation that the huge increase in the amount of complex non-proteincoding RNAs, which can represent up to $97-98 \%$ of all transcriptional output from genome (Mattick and Gagen, 2001; Mattick, 2003), it was deduced that the vast majority of the expressed information in the higher eukaryotes is in RNA, not proteincoding sequences. Taking also into account that very small (less than 1\%) of the sequence differences between individual humans occurs in protein coding sequences, a consequence should be that actually the majority of phenotypic variation between individuals (and species) results from differences in the control architecture, not in the proteins themselves. Indeed, the evidence is accumulating data showing that the major advance in the evolution of complex organisms was the co-option of RNA as a digital signaling network, which was required to overcome the limitations of an analog-type (protein) based regulatory system (Mattick and Makunin 2005). According to this report, the simple connection networks, such as telephone exchanges or the Internet, are able to grow in an unconstrained way. In contrast, regulatory networks -- such as those in biology (for example, the network of regulatory proteins that controls gene expression in bacteria), engineering, or society - are accelerating networks that must be able to operate in a globally responsive way (Mattick and Gagen, 2005). Therefore, it is to be pointed out that systems that require integral organization, like the human structures, in order to function in a competitive environment are dependent on, and ultimately constrained by, their accelerating regulatory architecture. Thus, connectivity and the proportion of the system devoted to regulation must scale faster than function in organized complex systems. On this requirement, the RNA networks are likely to be the most appropriate and intrinsically robust system solved by nature. The biology it has been used this network control architecture as a solution like a complex information processing system, to generate phenotypic complexity and variability (Mattick and Gagen, 2001).

From the above presentation it could be concluded that the cellular signaling and feed-back response are some of main pathway allowing the communication of different parts of the body, either by electric or non-electric signals. These signals carry the information to cells and inside of the cell by means of information embodiment/disembodiment mechanisms. It should be pointed out also that the complex functions in the high level organisms like the human body could be modeled and understood by using the tools and analogy with the processes approached by the science and technology of information.

\subsection{Phenomenological consequences added to IMC}

A main conclusive idea from the above presentation is that the increasing effort and successful compilation with the informational systems for processing of information by microelectronic means, from some simple circuits to informational network communication systems like telephony and Internet, and more suitable by "accelerated networks" (Mattick and Gagen, 2005), allows to describe and understand the functions and communication in the living structures at the molecular and cellular level from the informational perspective. However, the investigation of such function at micro-scale has to be regarded as a part of an integrated network communication and inter-change informational systems, in order to understand the macro-level behavior, taking into account that the functions at this levels are oriented to a common scope, that of flourishing and survival of the organism as a whole (Grandpierre et al., 2013). The collaboration of the biology, neuroscience and the science of information and technology may offer a suitable support for these topics. 
NeuroQuantology | April 2019| Volume 17 | Issue 04 | Page 01-17| doi: 10.14704/nq.2019.17.04.2009

Gaiseanu F., The Informational Model of Consciousness: Mechanisms of Embodiment/Disembodiment of Information

A first consequence revealed by the analysis presented above is that the communication between the cell implies a transmission agent and a specific receiver at the surface of the target cell. This type of communication was demonstrated to be an implied mechanism of "drug" type dependence (Dispenza, 2007). If a certain emotional state is insistently induced, the specific ligand and complementary receptor on the surface will establish a stable pair of promoters of the same emotion (or state), because the body cells themselves could "ask" the same type of emotions, if they are lived repetitively (Gaiseanu, 2018c). This is a feed-back cellular mechanism (Dispenza, 2007), like a drug dependence (Gupta and Kulhara, 2007). Within IMC, we define such type of induced emotional states as emotional long-range states, or simply, emo-states.

From the perspective of such an accumulated database, itcan be observed that IMC is able to describe the organism as a system of integration of information by embodiment/disembodiment mechanisms. A first level of integration is the capture of information, by IC and CASI. According to increasingly more literature, the epigenetics mechanisms are responsible for the information acquirement in the memory (CASI). The investigation of such mechanisms reveals that while the short-time memory is labile and instable, the long-time memory and the memory recovery are deeply associated with the epigenetic mechanisms in the nervous cells. These discoveries deeply support the earlier observations, showing that the repetition process reinforces the information acquirement, constituting a deeper step of a stable and observable embodiment of the information in the brain. The associated emotions could strongly participate to such a fixation process, as a body (cellular) reaction of the received information. In a subsequent step of a stable integration of information, an insistent repetitive process could integrate the information in automatic chains. This process could be expressed/ manifested either by fixed thinking or info-reaction patterns, which could be triggered by the same type of input information, becoming typical prototypes. The deeply exercised prototypes memorized by the "mother" cells could be transmitted to the offspring by means of epigenetic processes.

Such an embodiment process of information, starting from the receipt information as a result of the interaction with environment, in a stable, transmissible form to the next generation, shows that: (1) the human body is a learning system, with a high capacity of adaptation; (2) the human body is actually an active informational system, able to transform the free receipted information, resulted from the interaction with the environment for adaptation, in an automatic info-prototype and finally in an epigenetic transmissible information, expressible through the acquired traits, by means of information embodiment/disembodiment mechanisms. In terms of matter-related information, we have to observe that this is referred to cellular mechanisms, which intimately encode the operational information and can transmit it in a stable form by reproduction, incorporating new acquired traits. That's way, on a matter-related information scale, as it is shown in Fig. 1, the information embodiment mechanism of a stable acquired information increases from IC and CASI to GTS and IGG, as it was already pointed out.

A special discussion should be done on IC system. According to IMC, IC is the gate of connection with "dark matter" field (Gaiseanu, 2016a; 2017b,c; 2018d), as it was shown for the first time by Gaiseanu (Gaiseanu, 2016a). Dark matter could be actually interpreted as antimatter, according to a recent model of the universe (including our solar system (Hajdukovic, 2013)), with anti-symmetric properties with respect to matter, allowing to explain within IMC the paranormal phenomena(Gaiseanu,2017b,2018d). As consciousness is an informational anti-entropic system, directed to sustain the living and survival (Gaiseanu, 2016a; 2017a), the specific associated phenomena could be accordingly understood, first of all the disembodiment of consciousness from the body. This is done in a coherent manner, as a consequence of the anti-entropic nature both of antimatter (dark matter) field and consciousness itself. Therefore, the following associated phenomena could be explained (Gaiseanu, 2016a; 2017b): regression to the infancy during NDEs (determined according to IMC by the revers time arrow of antimatter field (Carroll, 2015)), the detection of a "tunnel"-like space, interpreted as the passing from a matter-bounded ("black-hole"-like) information to a ("luminous") mass-free informational entity in an anti-entropic (coherency) field (Gaiseanu, 2017b; $2018 \mathrm{~d}$ ). The extra-corporal view is a consequence of a direct "scanning" of the informational field of matter, by the own specific "mind' eye." The same power could explain also the remote diagnostics and the remote "view" of various geographic landscapes by means of the mind, as described earlier (Gaiseanu, 2018d). These phenomena show that the mind is, 
NeuroQuantology | April 2019| Volume 17 | Issue 04 | Page 01-17| doi: 10.14704/nq.2019.17.04.2009

Gaiseanu F., The Informational Model of Consciousness: Mechanisms of Embodiment/Disembodiment of Information

or could be connected to matter/anti-matter field, so to the informational field of the universe. The disembodiment seems to be a natural process, as a reverse phase of the information embodiment during the life-time cycle. If this is true, then an "after-life" phase of the disembodied consciousness entity could exist (Gaiseanu, 2018a). The time scale and the duration of such an entity is however an open question. IMC allows to approach on this way the immortality of the human being (Meijer 2013b; Hameroff and Chopra 2014) as a consequence of the disembody process (Gaiseanu 2018a).

Other consequences could be moreover discussed as follows. As it was suggested recently (Verlinde, 2010), the universal gravitational attraction between bodies could be deduced taking into account that information of a body is distributed on a surface (for instance of a spherical form) around each body, defined earlier as the "horizon of events" associated to a black-hole. As this should be valid for any kind of body, for the living structures this should be valid too. If this is true, then we can admit that the existence of an informational field, detected by some initiated persons as a "bio-field" or "aura" around the human body as described earlier (Kafatos et al., 2015), could be a valid issue of discussion, contradicting some sceptic opinions on this matter (Stenger, 1999).

From the perspective of the above discussion, consciousness could be defined as an integrated, dynamic and reactive state of connection and exploration of reality by detection, storing, operating, informational processing and decision making, expressed through attitude, with effects inside and/ or outside of the body, to adapt to the requirements of own organism and of the environment stimuli, for its survival. Consciousness is mainly manifested by data accumulation (Ik), informational operability (Iw), emotional reactivity (Il), functional self-control (Ia), associativity and creativity (Ic), self-confidence (Ib), on the basis of the genetic inheritance of species, received from the parents (Icd).

Connection and adaptation to the environment conditions is a dynamic informational process, which involves the integration of information into the operative and functional informational system of the bodythrough epigeneticmechanisms ofembodiment/ disembodiment of information. This process allows the long-term acquisition of new attributes, expressed by traits, which can be transmitted to the next generations. The human organism appears therefore as a global informational processor for adaptation and survival, embodying the convenient information (info-input) and disembodying information (infooutput) as attitude, info-genetic or out-body consciousness entity. This behavior demonstrates the high degree of intervention of information in the evolution of the body, determining its current and subsequent functional development, along one or more generations, under the form of matter-related information. Consciousness is enriched by the life experience and is maintained as a separated entity by a natural disembodiment process of information during the death of the organism. Therefore, the life cycle appears as an embodiment/disembodiment of information process into/from the matter structure.

\section{Conclusions}

It was shown that the informational system of the human body consists in seven informational subsystems, defined by IC (information pole connection), mainly responsible for the anti-entropic control of mind and of body, CASI, represented by memory, CDC - synthetizing the operative decisional and command informational unit, IES - expressed by emotions and emo-states, which mainly derives from the body reaction to information, MIS - mainly managing the matter connection and distribution in the body (matter-related pole), GTS - controlling the genetic transmission and IGG, which acts as a genetic informational input inherited from the two parents. For a complete description of IMC from the neuroscience perspective, the associated area in the brain corresponding to each defined subsystem, where the informational activity is promoted, were concretely specified. This informational neurodynamic system could be compared with the chakras empirical system known from the ancient oriental philosophies, opening a large gate for further investigations on this issue. The human body appears according to such a description as a binary info-matter system, connected to information (by the informational pole IC) and to matter, as a basic substrate supporting/incorporating (embodying) information.

These informational subsystems are reflected in consciousness by seven corresponding cognitive centers, suggestively called Ibelieve (Ib), Iknow (Ik), Iwant (Iw), Ilove (Il), Iam (Ia), Icreate (Ic) and Icreated (Icd), which define respectively the protection and trust, the library data as a life experience, the decision 
NeuroQuantology | April 2019| Volume 17 | Issue 04 | Page 01-17| doi: 10.14704/nq.2019.17.04.2009

Gaiseanu F., The Informational Model of Consciousness: Mechanisms of Embodiment/Disembodiment of Information

(mainly the attitude), the emotions and emo-states, the self status and health, and the predispositions and inherited traits. The info-connection of the body by means of IC with the outside environment can explain the paranormal phenomena.

To obtain the full panorama of the info-relation not only with the external but also with the internal communication and the implied mechanisms, the IMC structure was used to analyze the info-connectivity with the body itself. On a scale representing the integration degree of the input (free) information, it was deduced that the info-integration of the matterrelated information gradually increases from the info-connection (IC) to the IG (info-genetic output) and IGG (info-genetic input), where the genetic information is fully embodied. To understand the intimate process of the transition of information from free to highly embodied matter-related information, were identified specific inter and intra mechanisms of info-matter cell communications, by means of epigenetic messengers, which can be analyzed and modeled by analogy or correlation with the informational concepts and systems in the science of information and technology. In such a way it was shown that the epigenetic mechanisms are deeply involved in the long-time memory process of information embodiment and in the genetic transmission of the acquired traits, without affecting the genetic info-baggage of species. On the basis of the discussed results, a definition of consciousness was formulated in terms of information.

On the light of these results, it appears that the human organism is actually a dynamic reactive informational system, actuating for/by embodiment/ disembodimentofinformation:itisborn/formedfrom embodied genetic information, acquires information during the life time cycle, transmits genetic and epigenetic acquired information to the offspring and it is naturally submitted to a disembodiment process, consisting in the separation of the "living" information (disembodied consciousness) from matter, which become a non-living structure.

The results of this research open new and large perspectives for further investigations concerning the info-matter relation, contributing to fully understand consciousness and the intimate correlation mechanisms at micro and macro scale with the body itself and with the environment during the adaptation process.

\section{Acknowledgment}

To my son Adrian and to my close supporters, from the past to present and for the future, as a living expression of the continuity of the information and life.

\section{References}

Aharonov Y, Popescu S, Rohrlich D, Skrzypczyk P. Quantum cheshire cats. New Journal of Physics 2013; 15(11): 113015.

Alberts B, Johnson A, Lewis J, Raff M, Roberts K, Walter P. Molecular Biology of the Cell, 5-th Edition, New York, Garland Science, 2008.

Asashima Makoto. A Comprehensive Approach to the Life Science, Division of Advanced Education in Sciences, 2nd Ed., Yodosha, Komaba Organization for Educational Excellence, College of Arts and Sciences, The University of Tokyo, 2010.

Baars BJ. A Cognitive Theory of Consciousness. Cambridge, MA: Cambridge University Press, 1988.

Baars BJ and Gage N. Cognition, Brain and Consciousness, $2^{\text {nd }}$ ed., USA, Academic Press (Elsevier Sequoia), 2013.

Barritt, GJ. Communication Within Animal Cells. Oxford, UK: Oxford Science Publications, 1992.

Bösch H, Steinkamp F, Boller E. Examining psychokinesis: the interaction of human intention with random number generators--a meta-analysis. Psychol Bull 2006; 132(4): 497523.

Brugnoli MP, Pesce G, Pasin E, Basile MF, Tamburin S, Polati E. The role of clinical hypnosis and self-hypnosis to relief pain and anxiety in severe chronic diseases in palliative care: a 2-year long-term follow-up of treatment in a nonrandomized clinical trial. Annals of palliative medicine 2017; 7(1): 17-31.

Carroll S. The Arrow of Time. www.proposterousuniverse.com and From Eternity to Here, the Quest for the Ultimate Theory of Time. GB: Oneworld Publication, 2015.

Cortini R, Barbi M, Caré BR, Lavelle C, Lesne A, Mozziconacci J, Victor JM. The physics of epigenetics. Reviews of Modern Physics. 2016 Apr 26;88(2):025002-025029.

Cover TM and Thomas JA. Elements of Information Theory. New York: John Wiley \& Sons, Inc., 1991.

Davies P. Universe from bit. In Information and the Nature of Reality: From Physics to Metaphysics, eds P. Davies and N. Gregersen (Cambridge: Cambridge University Press), 2010.

Dehaene S, Charles L, King JR and Marti S. Toward a computational theory of conscious processing Current Opinion in Neurobiology 2014; 25: 76-84.

Dodd IB, Micheelsen MA, Sneppen K, Thon G. Theoretical analysis of epigenetic cell memory by nucleosome modification. Cell 2007; 129(4), 813-822.

Draganescu M. Informatia materiei (in Romanian)-Information of Matter. Bucharest: Romanian Academy, 1990.

Dulcan DC, Inteligenta Materiei (Intelligence of Matter), Ed. EIKON (Romania), 2009. 
NeuroQuantology | April 2019| Volume 17 | Issue 04 | Page 01-17| doi: 10.14704/nq.2019.17.04.2009

Gaiseanu F., The Informational Model of Consciousness: Mechanisms of Embodiment/Disembodiment of Information

Faria LCB, Rocha ASL, Palazzo R Jr. Transmission of intra-cellular genetic information: A system proposal. Journal of Theoretical Biology 2014; 358: 208-231.

Fracasso $\mathrm{C}$ and Friedman H. Near-Death Experiences and the Possibility of Disembodied Consciousness: Challenges to Prevailing Neurobiological and Psychosocial Theories. NeuroQuantology 2011; 9(1): 41-53.

Furusawa C and Kaneko K. Epigenetic Feedback Regulation Accelerates Adaptation and Evolution. PLoS ONE 2013; 8(5): e61251.

Gaiseanu F. Consciousness as Informational System of the Human Body. Consciousness and Life Physics, Cosmology and Astrophysics Journal 2016a; 16(1): 14-25.

Gaiseanu F. Informational Subsystems of the Consciousness. The Science of Consciousness, Abstract Book, TSC 2016 TUCSON. Ed. The University of Arizona Center for Consciousness Studies and University of Michigan Center for Consciousness Science, by Stuart Hameroff (The University of Arizona) and George Mashour (University of Michigan). 2016b. http:// www.consciousness.arizona.edu/documents/TSC2016_ B00K_of_Abstracts_for_emailWeb.pdf

Gaiseanu F. Quantum-Assisted Process of Disembody Under Near-Death Conditions: An Informational-Field Support Model." NeuroQuantology 2017a; 15(1): 4-9.

Gaiseanu F. An Information Based Model of Consciousness Fully Explaining the Mind Normal/Paranormal Properties. NeuroQuantology 2017b; 15.2: 132-140.

Gaiseanu F. Near-Death Experiences and Immortality from the Perspective of an Informational Modeling of Consciousness. Gerontology \& Geriatric Studies 2018a; 2(3): 1-4

Gaiseanu F. An Informational Modeling of Consciousness and Cognitive Centers. Proc. of the Human Project Brain (HPB) International Conference: Understanding Consciousness, a Scientific Quest for the 21st Century. 04-Models, simulation and emulation of consciousness, 2018b; Barcelona: 21-22 June.

Gaiseanu F. Destiny or Free Will Decision? A Life Overview From the Perspective of an Informational Modeling of Consciousness Part II: Attitude and Decision Criteria, Free Will and Destiny. Gerontology \& Geriatric Studies 2018c; 4(1): 1-7.

Gaiseanu F. Information: from Phylosophic to Physopics Concepts for Informational Modeling of Consciousness, Philosophy Study 2018d; 8(8): 368-382.

Gaiseanu F \& Graur A. Cognitive Centers Related Attitude: Application for an Iterative Evaluation Method in MusicBased Therapy Process. Abstract Book of the Conference on Science of Consciousness (Co-Chair Prof. Stuart Hameroff, The University of Arizona-Center for Consciousness Studies, Tucson, Arizona). Consciousness and Education-Cognitive Development, Concurrent Session C27, 165; 2018: April 2-7.

Gaiseanu F. Destiny or Free Will Decision? A Life Overview from the Perspective of an Informational Modeling of Consciousness Part I: Information, Consciousness and Life Cycle. Gerontology \& Geriatric Studies 2019a; 4(1): 1-7.

Gaiseanu F. Language Patterns and Cognitive-Sentient Reality: Certainty/Uncertainty in Cognitive-Sentient Exploration of Reality, Chap in Media Models to Foster Collective Human
Coherence in the PSYCHecology, Ed. Stephen Brock Schafer, USA, IGI Global, 2019b (in press).

Gardiner J. The Fractal Nature of the Brain: EEG Data Suggests That the Brain Functions as a Quantum Computer in 5-8 Dimensions. NeuroQuantology 2010; 8(2): 137-141.

Gawad J, Chavan B, Bawane P, Mhaske A, Tauro S. Overview of Cell Signaling and Cell Communication. Journal of Pharmaceutical Biology 2015; 5(2): 104-107.

Gorski D. Epigenetics: it doesn't mean what quacks think it means, 2013: https://sciencebasedmedicine.org/epigeneticsit-doesnt-mean-what-quacks-think-it-means /

Grandpierre A, Chopra D, Doraiswamy MP, Tanzill R, Kafatos MC. A Multidisciplinary Approach to Mind and Consciousness. NeuroQuantology 2013; 11(4)4: 607-617.

Gupta S and Kulhara P. Cellular and molecular mechanisms of drug dependence: An overview and update, Indian J Psychiatry 2007; 49(2): 85-90.

Hajdukovic D. Can Observations Inside the Solar System Reveal the Gravitational Properties of the Quantum Vacuum? Astrophys Space Sci. 2013; 343: 505-509.

Hameroff S. Quantum Computation in Brain Microtubules? The Pennrose-Hameroff. 'Orch OR' Model of Consciousness. Philos. Trans. Royal Society London, Ser. A, Math. Phys. Sci. 1998; 356: 1869-98.

Hameroff S and Chopra D. Can Science Explain the Soul?. 2010; 2014. https://www.huffingtonpost.com/deepak-chopra/canscience-explain-the-s_b_675107.html?guccounter=1.

Hameroff S \&Penrose R. Consciousness in the Universe: A Review of the 'Orch. OR' Theory. Physics of the Life Review 2014; 1: 39-79.

Herholz SC, Coffey EBJ, Pantev H, and Zatorre RJ. Dissociation of Neural Networks for Predisposition and for Training-Related Plasticity in Auditory-Motor Learning, Cerebral Cortex 2016; 26: 3125-3134.

Hines T. Anatomy of the Brain. Mayfield Brain \& Spine, 2018. https://mayfieldclinic.com/pe-anatbrain.htm.

Ignatenko A. Cum sa devii un fenomen (in Romanian)-How to Become a Phenomenon. Bucharest: Biodova, 1994.

Inzlicht M, Tullett A, Good M. The need to believe: a neuroscience account of religion as a motivated process, Religion, Brain \& Behavior 2011;1 (3): 192-251.

Jarome JT and Lubin FD. Epigenetic Mechanisms of Memory Formation and Reconsolidation. Neurobiol Learn Mem 2014; $0: 116-127$.

Kafatos MC, Chevalier G, Chopra D, Hubacher J, Kak S. Biofield Science: Current Physics Perspectives Neil D. Theise, Global Adv Health Med. 2015; 4(suppl): 25-34.

Kaku M. The Future of the Mind, The Scientific Quest to Understand Enhance and Empowered the Mind, NY, London, Toronto, Sidney Aukland: Doubleday, Division of Random House Canada, Toronto, Penguin Random Hose Companies, Twin Revolution, 2014.

Laub MT. Keeping Signals Straight: How Cells Process Information and Make Decisions. PLoS Biol 2016; 14(7): e1002519. 
NeuroQuantology | April 2019| Volume 17 | Issue 04 | Page 01-17| doi: 10.14704/nq.2019.17.04.2009

Gaiseanu F., The Informational Model of Consciousness: Mechanisms of Embodiment/Disembodiment of Information

Levenson JM and Sweatt JD. Epigenetic Mechanisms in Memory Formation. Nature Reviews, Neuroscience 2005; 6: 108-118

Lubin FD, Gupta S, Parrish RR, Grissom NM, Davis RL. Epigenetic mechanisms: critical contributors to long-term memory formation. Neuroscientist 2011; 17(6): 616-632.

Mattick JS. Challenging the dogma: the hidden layer of nonprotein-coding RNAs in complex organisms. BioEssays W003 2003; 25: 930-939.

Mattick JS and Gagen JM. The Evolution of Controlled Multitasked Gene Networks: The Role of Introns and Other Noncoding RNAs in the Development of Complex Organisms. Mol Biol Evol 2001; 18: 1611-1630.

Mattick JS and Gagen JM. Accelerating Networks, Science 2005; 307: 856-858.

Mattick JS and Makunin IV. Small regulatory RNAs in mammals. Human Molecular Genetics 2005; 14(1): R121-R132.

Meijer D. Information: What Do You Mean? On the Formative Element of Our Universe. Syntropy 2013a; 3: 1-49.

Meijer D. Immortality: Myth or Becoming Reality? On the Conservation of Information. Syntropy 2013b; 3: 168-207.

Myss C. Anatomy of the Spirit-The Seven Stages of Power and Healing. USA: Crown Publishers, Inc., 1996.

Odoul M. Ghidul complet al durerilor fizice-Terapii psihoenergetice, Prefață Dr. Medynski, Thierry (in Romanian, translated from Franch)-The Complete Physical Pain Guide-Psycho-Therapeutic Therapies, Preface by Dr. Medynski, Thierry. Bucuresti: Ed. Paralela 45, 2014. https:// caleaechilibruluii.files.wordpress.com/2014/04/michelodoul-ghidul-complet-al-durerilor-fizice.pdf.

Overgaard M. The Status and Future of Consciousness Research. SPECIALTY GRAND CHALLENGE, Frontiers in Psychology 2017; 8: 1-4.

Pepperell R. Consciousness as a Physical Process Caused by the Organization of Energy in the Brain. Front.Psychol 2018; 9: $1-11$.

Perlovski L. Neural Networks and Intellect: Using Model Based Concepts. Oxford: University Press, 2001.

Radin D. Entangled Minds, Extrasensory Experiences in a Quantum Theory. NY, London, Toronto, Sydney: Paraview Pocket Books, a division of Simon Shuster, Inc., 2006.

Radin D. Real Magic, Ancient Wisdom, Modern Science, and a Guide to the Secret Power of the Universe. New York: Harmony Books, an imprint of the Crown Publishing Group, a division of Penguin Random House LLC, 2018.
Rae P. Into the Psychokinetic (Pk) Zone: A Phenomenological Study of the Experience of Performing Pk. The Journal of Parapsychology 1999; 63(3): 112-122.

Ruffini, G. An algorithmic information theory of consciousness, Neurosci. Conscious. 2017; 3: nix019.

Satsangi AK and Brugnoli MP. Anxiety and psychosomatic symptoms in palliative care: from neuro-psychobiological response to stress, to symptoms' management with clinical hypnosis and meditative states. Annals of Palliative Medicine 2018; 7(1): 75-111.

Shan L. Consciousness is an Entity with Entangled States: Correlating the Measurement Problem with Non-Local Consciousness.NeuroQuantology 2018; 16(7): 70-78.

Shannon C. The Mathematical Theory of Communication. Bell Syst Tech J 1948; 27: 379-423.

Shelley L, Berger LS, Kouzarides T, Shiekhattar R, Shilatifard A. An operational definition of epigenetics, Genes Dev 2009; 23(7): 781-783.

Sogin ML, Gunderson JH, Elwood HJ, Alonso RA, Peattie DA. Alberts Molecular Biology Albert Paginas (PubMed), 2003.

Stenger VJ. Bioenergetic Fields. The Scientific Review of Alternative Medicine 1999; 3(1).

Tegmark M. Our Mathematical Universe: My Quest for the Ultimate Nature of Reality. USA: Random House, 2014.

Tononi G. "An Information Integration Theory of Consciousness." BMC Neuroscience 2004; 5: 42.

Tononi G. Consciousness as Integrated Information: A Provisional Manifesto. Biol. Bull. 2008; 215: 216-242.

Tononi, G., Boly, M., Massimini, M., and Koch, C. Integrated information theory: from consciousness to its physical substrate. Nat. Rev. Neurosci 2016; 17: 450-461.

Van Lommel P. Near-Death Experience, Consciousness, and the Brain: A New Concept About the Continuity of Our Consciousness Based on Recent Scientific Research on NearDeath Experience in Survivors of Cardiac Arrest. World Futures 2006; 62: 134-151.

Verlinde E. On the Origin of Gravity and the Laws of Newton. University of Amsterdam Valckenierstraat 2010; 65.

Woldemichael BT and Mansuy IM. Epigenetic Basis of Memory. In Sara SJ, Brian JH, Learning and memory: a comprehensive reference, Amsterdam, Elsevier 2017; pp:247-256.

Zhang Y, Kim MS, Jia B, Yan J, Zuniga-Hertz PJ, and Han C, Hypothalamic stem cells control ageing speed partly through exosomal miRNAs. Nature 2017; 548: 52-57. 